\title{
ОПТИМАЛЬНОЕ ВОССТАНОВЛЕНИЕ АНАЛИТИЧЕСКОЙ В ПОЛУПЛОСКОСТИ ФУНКЦИИ ПО ПРИБЛИЖЕННО ЗАДАННЫМ ЗНАЧЕНИЯМ НА ЧАСТИ ГРАНИЧНОЙ ПРЯМОЙ ${ }^{1}$
}

\author{
P. Р. Акопян
}

\begin{abstract}
Пусть $\mathcal{H}^{p}\left(\Pi_{+}, \phi\right)-$ класс аналитических в верхней полуплоскости $\Pi_{+}$функций, принадлежащих универсальному классу Харди $N_{*}$, с граничными значениями из $L_{\phi}^{p}(\mathbb{R})$ с весом $\phi ; Q^{p}\left(\Pi_{+}, \mathbb{I}, \phi\right)-$ класс функций $f \in \mathcal{H}^{p}\left(\Pi_{+}, \phi\right)$ таких, что $\|f\|_{L_{\phi}^{p}(\mathbb{R} \backslash \mathbb{I})} \leq 1$, где $\mathbb{I}-$ промежуток (интервал или полупрямая) из $\mathbb{R}$, $1 \leq p \leq \infty$. На классе $Q^{p}\left(\Pi_{+}, \mathbb{I}, \phi\right)$ в задаче оптимального восстановления значения функции в точке $z_{0} \in \Pi_{+}$по ее приближенно заданным предельным граничным значениям на $\mathbb{I}$ по норме $L_{\phi}^{p}(\mathbb{I})$ и взаимосвязанной задаче наилучшего приближения функционала линейными ограниченными функционалами явно выписаны решения - экстремальная функция, оптимальный метод восстановления, функционал наилучшего приближения. На классе $Q^{p}\left(\Pi_{+}, \mathbb{R}_{+}, \psi\right), \psi(z)=1 /|z|$, решены задача оптимального восстановления функции на луче $\gamma=\left\{z: \arg z=\varphi_{0}\right\}$ относительно нормы $L_{\psi}^{p}(\gamma)$ по ее приближенно заданным предельным граничным значениям на $\mathbb{R}_{+}$по норме $L_{\psi}^{p}\left(\mathbb{R}_{+}\right)$и взаимосвязанная задача наилучшего приближения оператора линейными ограниченными операторами. Для $f \in \mathcal{H}^{p}\left(\Pi_{+}, \psi\right)$ получено точное неравенство
\end{abstract}

$$
\|f\|_{L_{\psi}^{p}(\gamma)} \leq\|f\|_{L_{\psi}^{p}(-\infty, 0)}^{\varphi_{0} / \pi}\|f\|_{L_{\psi}^{p}(0,+\infty)}^{1-\varphi_{0} / \pi}
$$

Ключевые слова: оптимальное восстановление оператора, наилучшее приближение неограниченного оператора ограниченными операторами, аналитические функции.

R. R. Akopyan. Optimal recovery of a function analytic in a half-plane from approximately given values on a part of the straight-line boundary.

Let $\mathcal{H}^{p}\left(\Pi_{+}, \phi\right)$ be the class of functions analytic in the upper half-plane $\Pi_{+}$and belonging to the universal Hardy class $N_{*}$ with boundary values from $L_{\phi}^{p}(\mathbb{R})$ with a weight $\phi$, and let $Q^{p}\left(\Pi_{+}, \mathbb{I}, \phi\right)$ be the class of function $f \in \mathcal{H}^{p}\left(\Pi_{+}, \phi\right)$ such that $\|f\|_{L_{\phi}^{p}(\mathbb{R} \backslash \mathbb{I})} \leq 1$, where $\mathbb{I}$ is a finite open interval or a half-line from $\mathbb{R}$ and $1 \leq p \leq \infty$. On the class $Q^{p}\left(\Pi_{+}, \mathbb{I}, \phi\right)$, we consider the problem of optimal recovery of the value of a function at a point $z_{0} \in \Pi_{+}$ from its approximately given limit boundary values on $\mathbb{I}$ in the norm $L_{\phi}^{p}(\mathbb{I})$ and the related problem of the best approximation of a functional by linear bounded functionals. Explicit solutions of these problems are written: an extremal function, optimal recovery method, and best approximation functional. On the class $Q^{p}\left(\Pi_{+}, \mathbb{R}_{+}, \psi\right)$, $\psi(z)=1 /|z|$, we solve the problem of optimal recovery of a function on a ray $\gamma=\left\{z: \arg z=\varphi_{0}\right\}$ with respect to the norm $L_{\psi}^{p}(\gamma)$ from its approximately given limit boundary values on $\mathbb{R}_{+}$in the norm $L_{\psi}^{p}\left(\mathbb{R}_{+}\right)$and the related problem of the best approximation of an operator by linear bounded operators. For $f \in \mathcal{H}^{p}\left(\Pi_{+}, \psi\right)$, we obtain the exact inequality

$$
\|f\|_{L_{\psi}^{p}(\gamma)} \leq\|f\|_{L_{\psi}^{p}(-\infty, 0)}^{\varphi_{0} / \pi}\|f\|_{L_{\psi}^{p}(0,+\infty)}^{1-\varphi_{0} / \pi}
$$

Keywords: optimal recovery of an operator, best approximation of an unbounded operator by bounded operators, analytic function.

MSC: 30A10, 30C80, 30C85, 30E10

DOI: $10.21538 / 0134-4889-2018-24-4-19-33$

\section{Введение}

Обозначим через $\Pi_{+}$верхнюю полуплоскость: $\Pi_{+}=\{z \in \mathbb{C}: \Im z>0\}$. Через $\mathbb{I}$ обозначим промежуток граничной прямой $\mathbb{R}$, в качестве которого будет выступать либо конечный интервал $(a, b)$, либо полупрямая $(0,+\infty)$.

\footnotetext{
${ }^{1}$ Работа выполнена при поддержке РФФИ (проект № 18-01-00336) и Программы повышения конкурентоспособности УрФУ (постановление № 211 Правительства РФ от 16.03.2013, контракт № 02.А03.21.0006 от 27.08.2013).
} 
Напомним некоторые известные понятия и факты, которые будут использоваться в статье. Для ядра Пуассона полуплоскости справедливо равенство

$$
P(z, \xi)=\Re\left\{\frac{1}{i \pi} \frac{1}{z-\xi}\right\}=\frac{1}{\pi} \frac{y}{(x-\xi)^{2}+y^{2}}, \quad z=x+i y, \quad \xi \in \mathbb{R} .
$$

Гармонической мерой $w(z)=w\left(z, \mathbb{I}, \Pi_{+}\right)$промежутка $\mathbb{I}$ относительно полуплоскости $\Pi_{+}$в точке $z \in \Pi_{+}$является решение задачи Дирихле - ограниченная гармоническая в $\Pi_{+}$функция с граничными значениями $w(t)=1, t \in \mathbb{I}$ и $w(t)=0, t \in \mathbb{R} \backslash \overline{\mathbb{I}}$. Для $w\left(z, \mathbb{I}, \Pi_{+}\right)$имеет место равенство

$$
w\left(z, \mathbb{I}, \Pi_{+}\right)=\frac{1}{\pi} \int_{\mathbb{I}} \frac{y}{(x-t)^{2}+y^{2}} d t .
$$

Соответственно для промежутков на прямой получим

$$
w\left(z,(a, b), \Pi_{+}\right)=\frac{1}{\pi} \arg \frac{z-b}{z-a} ; \quad w\left(z,(0,+\infty), \Pi_{+}\right)=\frac{\pi-\arg z}{\pi} .
$$

Классом Неванлинны $N=N\left(\Pi_{+}\right)$называют множество аналитических в $\Pi_{+}$функций $f$, для которых субгармоническая функция $\ln ^{+}|f|$ имеет в $\Pi_{+}$гармоническую мажоранту. Функции класса $N$ имеют почти всюду на $\mathbb{R}$ некасательные предельные граничные значения. Более узкий класс $N_{*}=N_{*}\left(\Pi_{+}\right)$- универсальный класс Харди, введенный В. И. Смирновым, состоит из функций, имеющих гармоническую мажоранту $\ln ^{+}|f|$, представимую по формуле Грина через ее граничные значения. Классом Харди $H^{p}=H^{p}\left(\Pi_{+}\right), 0<p<\infty$, называют класс функций $f$, для которых $|f|^{p}$ имеет гармоническую мажоранту в $\Pi_{+} ;$при $p=\infty$ класс $H^{\infty}=H^{\infty}\left(\Pi_{+}\right)$- класс ограниченных аналитических функций. Справедливы вложения $H^{p} \subset N_{*} \subset N$. Функция $f \in N_{*}$ принадлежит классу Харди $H^{p}$ тогда и только тогда, когда ее граничные значения удовлетворяют условию: функция $|f|^{p}$ суммируема на прямой $\mathbb{R}$ с весом $P\left(z_{0}, x\right)$, где $z_{0}$ - произвольная фиксированная точка из $\Pi_{+}$. Аналитическая в полуплоскости $\Pi_{+}$функция представима через свои граничные значения по формуле Грина, т.е. справедливо представление

$$
f(z)=\int_{-\infty}^{+\infty} P(z, x) f(x) d x, \quad z \in \Pi_{+},
$$

тогда и только тогда, когда она принадлежит классу Харди $H^{1}\left(\Pi_{+}\right)$.

Далее будут рассматриваться класс $\mathcal{H}=\mathcal{H}^{p}\left(\Pi_{+}, \phi\right), 1 \leq p \leq \infty$, функций $f \in N_{*}$ с граничными значениями из $L_{\phi}^{p}(\mathbb{R})$ с весом $\phi$ и подкласс $Q=Q^{p}\left(\Pi_{+}, \mathbb{I}, \phi\right)$ функций $f \in \mathcal{H}$ таких, что $\|f\|_{L_{\phi}^{p}(\mathbb{R} \backslash \mathbb{I})} \leq 1$.

В первой части статьи рассмотрены взаимосвязанные задачи оптимального восстановления и наилучшего приближения на классе $Q^{p}\left(\Pi_{+}, \mathbb{I}, \phi\right)$ функционала, сопоставляющего предельным граничным значениям на промежутке (интервале или полупрямой) функции, аналитической в полуплоскости, ее значение во внутренней точке. Более общая постановка (для широкого класса областей и норм) исследовалась в терминах геометрической теории функции комплексного переменного в работах автора [3;4]. Однако явный аналитический вид решения этих задач (экстремальные функции и функционалы, константы точных неравенств) удается выписать в редких случаях. Именно такой случай рассмотрен здесь.

Во второй части на классе $Q^{p}\left(\Pi_{+}, \mathbb{R}_{+}, \psi\right), \psi(z)=1 /|z|$, решены задачи оптимального восстановления и наилучшего приближения оператора, ставящего в соответствие предельным граничным значениям функции на полупрямой ее сужение на луч.

Явный вид функционала (оператора), для которого рассматриваются задачи оптимального восстановления и наилучшего приближения, дают формулы Карлемана - Голузина Крылова. Задача вычисления этого функционала (оператора) по заданным с погрешностью 
граничным значениям является некорректной, методы ее регуляризации были предложены М. М. Лаврентьевым. Информацию о формулах Карлемана-Голузина-Крылова и методе Лаврентьева можно найти в [ 1, гл. 1; 12, гл. II, §1, пп. 4, 5].

Рассмотренные в статье задачи являются частными случаями, соответственно, задачи оптимального восстановления операторов на классе элементов банахова пространства по неполной (в частности, неточной) информации и задачи Стечкина о приближении неограниченного оператора ограниченными операторами на классе элементов банахова пространства. Общие результаты в тематике оптимального восстановления и дальнейшие ссылки можно найти в [6-8; 13-15;19]. Результаты, связанные с оптимальным восстановлением на классах аналитических функций, и дальнейшие ссылки можно найти в монографии [16] и в работах $[3 ; 5 ; 10 ; 11 ; 17 ; 18]$. Задаче Стечкина к настоящему времени посвящено большое число исследований (см. работы $[8 ; 9]$ и приведенную в них библ.).

В дальнейшем $\ln z=\ln |z|+i \arg z, 0 \leq \arg z<2 \pi$, и $z^{\zeta}=\exp \{\zeta \ln z\}, z, \zeta \in \mathbb{C}, z \neq 0$.

\section{1. Оптимальное восстановление значения}

\section{в точке аналитической в полуплоскости функции по приближенно заданным на промежутке граничным значениям}

На классе $Q$ рассмотрим взаимосвязанные экстремальные задачи для функционала $\Upsilon_{z_{0}}=$ $\Upsilon_{z_{0}}\left(\mathbb{I}, \Pi_{+}\right)$, сопоставляющего граничным значениям на $\mathbb{I}$ аналитической функции ее значение в точке $z_{0} \in \Pi_{+}$, т. е. $\Upsilon_{z_{0}} f=f\left(z_{0}\right)$. Функцию переменной $\delta>0$, определяемую равенством

$$
\omega(\delta)=\omega\left(\delta ; \Upsilon_{z_{0}}, Q\right):=\sup \left\{\left|f\left(z_{0}\right)\right|: f \in Q,\|f\|_{L_{\phi}^{p}(\mathbb{I})} \leq \delta\right\}
$$

называют модулем непрерывности функиионала $\Upsilon_{z_{0}}$ на классе $Q$. Из определения (1.1) для функций $f$ из $\mathcal{H}$ следует точное неравенство

$$
\left|f\left(z_{0}\right)\right| \leq\|f\|_{L_{\phi}^{p}(\mathbb{R} \backslash \mathbb{I})} \omega\left(\frac{\|f\|_{L_{\phi}^{p}(\mathbb{I})}}{\|f\|_{L_{\phi}^{p}(\mathbb{R} \backslash \mathbb{I})}}\right), \quad f \in \mathcal{H} .
$$

С задачей (1.1) о модуле непрерывности связана задача оптимального восстановления значения аналитической в $\Pi_{+}$функции в точке $z_{0}$ (функционала $\Upsilon_{z_{0}}$ ) по заданным с известной погрешностью $\delta$ по норме $L_{\phi}^{p}(\mathbb{I})$ ее граничным значениям на $\mathbb{I}$ и дополнительной (априорной) информации принадлежности функции классу $Q$. Более точно, пусть для неизвестной функции $f$ из класса $Q$ задана функция $\widetilde{f} \in L_{\phi}^{p}(\mathbb{I})$ такая, что справедливо неравенство $\|f-\widetilde{f}\|_{L_{\phi}^{p}(\mathbb{I})} \leq \delta$. Мы хотим найти наилучший (оптимальный) способ восстановить по $\tilde{f}$ значение функции $f\left(z_{0}\right), z_{0} \in \Pi_{+}$, для всех таких пар функций $f$ и $\widetilde{f}$. В качестве множества методов восстановления, из которых выбирается оптимальный, будем рассматривать множество $\mathcal{O}$ всех возможных функционалов на $L_{\phi}^{p}(\mathbb{I})$. Формальная постановка задачи такова. Для числа $\delta \geq 0$ и метода восстановления $T \in \mathcal{O}$ величина

$$
\mathcal{U}(T, \delta):=\sup \left\{\left|f\left(z_{0}\right)-T \widetilde{f}\right|: f \in Q, \widetilde{f} \in L_{\phi}^{p}(\mathbb{I}),\|f-\widetilde{f}\|_{L_{\phi}^{p}(\mathbb{I})} \leq \delta\right\}
$$

является погрешностью восстановления значения в точке $z_{0}$ функций класса $Q$ по их граничным значениям на $\mathbb{I}$, заданным с ошибкой $\delta$ по норме $L_{\phi}^{p}(\mathbb{I})$, методом $T$. Тогда

$$
\mathcal{E}(\delta):=\inf \{\mathcal{U}(T, \delta): T \in \mathcal{O}\}
$$

есть величина оптимального восстановления значения в точке $z_{0}$ (или, что то же самое, оптимального восстановления функционала $\Upsilon_{z_{0}}$ ) функций класса $Q$ по их $\delta$-приближенным граничным значениям на II с помощью всех возможных методов восстановления. Задача состоит 
в вычислении величины $\mathcal{E}(\delta)$ и определении оптимального метода восстановления - функционала, на котором в (1.4) достигается нижняя грань. Известно (см., например, $[7 ; 8 ; 13 ; 16]$ и приведенную там библ.), что в задаче оптимального восстановления линейного функционала на выпуклом центрально симметричном классе с помощью множества $\mathcal{O}$ всех возможных функционалов существует наилучший линейный ограниченный функционал и сама величина уклонения равна модулю непрерывности восстанавливаемого функционала, таким образом справедливо равенство $\mathcal{E}(\delta)=\omega(\delta)$.

С задачами (1.1) и (1.4) тесно связана задача наилучшего приближения функционала $\Upsilon_{z_{0}}$ линейными ограниченными функционалами. Точная постановка задачи такова. Пусть $\mathcal{L}(N)$ есть множество линейных ограниченных функционалов на $L_{\phi}^{p}(\mathbb{I})$, норма которых не превосходит числа $N>0$. Величина

$$
U(T):=\sup \left\{\left|f\left(z_{0}\right)-T f\right|: f \in Q\right\}
$$

является уклонением функционала $T \in \mathcal{L}(N)$ от функционала $\Upsilon_{z_{0}}$ на классе функций $Q$. Наилучшим приближением функционала $\Upsilon_{z_{0}}$ множеством линейных ограниченных функционалов $\mathcal{L}(N)$ на классе $Q$ называется величина

$$
E(N):=\inf \{U(T): T \in \mathcal{L}(N)\} .
$$

Задача состоит в вычислении величины $E(N)$ и нахождении экстремального функционала, на котором в (1.6) достигается нижняя грань.

Для формулировки результатов в задачах (1.4) и (1.6) приведем конструкции некоторых функций и функционала. Зададим функцию $h_{\mathbb{I}}$ в полуплоскости $\Pi_{+}$равенством $h_{\mathbb{I}}(z):=$ $\exp \{w(z)+i \widetilde{w}(z)\}$, в котором $w(z)=w\left(z, \mathbb{I}, \Pi_{+}\right)$- гармоническая мера промежутка $\mathbb{I}$ относительно полуплоскости $\Pi_{+}$в точке $z, \widetilde{w}-$ функция гармонически сопряженная $w$, которая определена и единственна, с точностью до аддитивной (вещественной) константы, выбор которой нам не важен. Функция $h_{\mathbb{I}}$ является ограниченной аналитической, не обращается в нуль в полуплоскости $\Pi_{+}$и удовлетворяет равенствам

$$
\left|h_{\mathbb{I}}(z)\right|=e^{w(z)}, \quad z \in \Pi_{+} ; \quad\left|h_{\mathbb{I}}(x)\right|=e, \quad x \in \mathbb{I} ; \quad\left|h_{\mathbb{I}}(x)\right|=1, \quad x \in \mathbb{R} \backslash \overline{\mathbb{I}} .
$$

Для фиксированной точки $z_{0} \in \Pi_{+}$определим функцию $f_{z_{0}}$ соотношением

$$
f_{z_{0}}(z):=\frac{y_{0}}{\pi} \frac{1}{\left(z-\overline{z_{0}}\right)^{2}}, \quad y_{0}=\Im z_{0} .
$$

Сужение модуля функции $f_{z_{0}}$ на вещественную ось совпадает с ядром Пуассона, т. е. $\left|f_{z_{0}}(x)\right|=$ $P\left(z_{0}, x\right), x \in \mathbb{R}$. Третья функция будет построена по весовой функции $\phi$. Пусть неотрицательная измеримая функция $\phi$ удовлетворяет условию: $\ln \phi$ является суммируемой функцией на $\mathbb{R}$ по гармонической мере, т. е. конечна величина

$$
\int_{-\infty}^{+\infty} \frac{|\ln \phi(x)|}{x^{2}+1} d x<+\infty .
$$

Аналитическую в полуплоскости $\Pi_{+}$функцию $g_{\phi}$ определим равенством $g_{\phi}(z):=\exp \{u(z)+$ $i \widetilde{u}(z)\}$, в котором гармоническая в $\Pi_{+}$функция $u$ является интегралом Пуассона

$$
u(z)=-\int_{-\infty}^{+\infty} P(z, x) \ln \phi(x) d x
$$

а $\widetilde{u}$ - функция, гармонически сопряженная $u$, для которой выбор аддитивной постоянной нам также не важен. Функция $g_{\phi}$ в полуплоскости в нуль не обращается. Граничные значения 
модуля функции $g_{\phi}$ почти всюду совпадают с функцией $1 / \phi$, т. е. $\left|g_{\phi}(x)\right|=1 / \phi(x)$ для почти всех $x \in \mathbb{R}$. Например, в случае "степенных" весов $\phi_{\nu}, \psi_{\nu}, \nu \in \mathbb{R}$, определяемых равенствами

$$
\begin{gathered}
\phi_{\nu}\left(z_{1}, x\right):=P^{\nu}\left(z_{1}, x\right)=\left(\frac{1}{\pi} \frac{y_{1}}{\left(x-x_{1}\right)^{2}+y_{1}^{2}}\right)^{\nu}, \quad z_{1}=x_{1}+i y_{1} \in \Pi_{+}, \\
\psi_{\nu}\left(x_{1}, x\right):=\left|x-x_{1}\right|^{-2 \nu}, \quad x_{1} \in \mathbb{R},
\end{gathered}
$$

функции $g_{\phi_{\nu}}$ и $g_{\psi_{\nu}}$ имеют вид

$$
g_{\phi_{\nu}}(z)=\left(y_{1} / \pi\right)^{-\nu}\left(z-\overline{z_{1}}\right)^{2 \nu}=f_{z_{1}}^{-\nu}(z), \quad g_{\psi_{\nu}}(z)=\left(z-x_{1}\right)^{2 \nu}, \quad z \in \Pi_{+} .
$$

Напомним, что для произвольной точки $z_{1} \in \Pi_{+}$и веса $\phi_{1}\left(z_{1}, x\right)$ класс $\mathcal{H}^{p}\left(\Pi_{+}, \phi_{1}\right)$ совпадает с классом Харди $H^{p}\left(\Pi_{+}\right)$.

Используя введенные выше функции $h_{\mathbb{I}}, f_{z_{0}}$ и $g_{\phi}$, определим важную в дальнейшем функцию $F$ формулой

$$
\begin{gathered}
F(z)=F\left[z_{0}, \mathbb{I}, p, \phi, \delta\right](z):=f_{z_{0}}^{1 / p}(z) g_{\phi}^{1 / p}(z) \beta^{-1 / p} h_{\mathbb{I}}^{\sigma}(z), \\
\sigma:=\ln \delta-1 / p \ln \alpha+1 / p \ln \beta, \quad \alpha:=w\left(z_{0}, \mathbb{I}, \Pi_{+}\right), \quad \beta:=1-\alpha=w\left(z_{0}, \mathbb{R} \backslash \mathbb{I}, \Pi_{+}\right)
\end{gathered}
$$

при $1 \leq p \leq \infty$. В случае $p=\infty$ величину $1 / p$ будем всегда считать равной нулю. Функция $F$ является аналитической и не обращается в нуль в полуплоскости П + . Для ее значений на вещественной оси справедливы равенства

$$
|F(x)|=\delta\left(\frac{P\left(z_{0}, x\right)}{\alpha \phi(x)}\right)^{1 / p}, \quad x \in \mathbb{I} ; \quad|F(x)|=\left(\frac{P\left(z_{0}, x\right)}{\beta \phi(x)}\right)^{1 / p}, \quad x \in \mathbb{R} \backslash \overline{\mathbb{I}}
$$

а для значения в точке $z_{0}-$ равенство

$$
\left|F\left(z_{0}\right)\right|=\mathcal{C} \delta^{\alpha}, \quad \mathcal{C}:=\left\{\frac{\alpha^{-\alpha} \beta^{-\beta}}{4 \pi y_{0}}\left|g_{\phi}\left(z_{0}\right)\right|\right\}^{1 / p} .
$$

Отметим, что функция $F$ принадлежит классу $\mathcal{H}$. Более того, используя равенства (1.9), нетрудно получить равенства

$$
\|F\|_{L_{\phi}^{p}(\mathbb{I})}=\delta, \quad\|F\|_{L_{\phi}^{p}(\mathbb{R} \backslash \mathbb{I})}=1
$$

следовательно, функция $F$ принадлежит классу $Q$.

Теперь на пространстве $L_{\phi}^{p}(\mathbb{I})$ определим функционал $T_{\delta}=T\left[z_{0}, \mathbb{I}, p, \phi, \delta\right]$ равенством

$$
T_{\delta} f:=\int_{\mathbb{I}} P\left(x, z_{0}\right) \frac{F\left(z_{0}\right)}{F(x)} f(x) d x .
$$

Теорема 1. Пусть $1 \leq p \leq \infty$, функиия ф удовлетворяют условию (1.7). Тогда справедливы следующие утверждения.

1) Для величин (1.1) и (1.4) имеют место равенства

$$
\omega(\delta)=\mathcal{E}(\delta)=\mathcal{C} \delta^{\alpha}, \quad \delta \geq 0 .
$$

Экстремальными в (1.1) являются функиии вида $\epsilon F,|\epsilon|=1$; в задаче (1.4) оптимальным методом восстановления является линейный ограниченный функиионал $T_{\delta}$.

2) Для величины (1.6) справедливо равенство

$$
E(N)=\mathcal{C}^{1 / \beta} \beta \alpha^{\alpha / \beta} N^{-\alpha / \beta} .
$$

В задаче (1.6) функиионалом наилучшего приближения является функиионал $T_{\delta}$ с параметром $\delta=\mathcal{C}^{1 / \beta} \alpha^{1 / \beta} N^{-1 / \beta}$.

Далее, с использованием равенств (0.1) и теоремы 1 будут выписаны экстремальные функция $F$ и функционал $T_{\delta}$, а также неравенство (1.2) в случаях, когда промежуток $\mathbb{I}$ является конечным интервалом $(a, b)$ или полуосью $(0,+\infty)$. 


\section{1. Экстремальные функция и функционал в случае $\mathbb{I}=(a, b)$}

В случае, когда промежутком $\mathbb{I}$ является интервал $(a, b)$, для гармонических мер имеем

$$
\alpha=w\left(z_{0},(a, b), \Pi_{+}\right)=\frac{\vartheta}{\pi}, \quad \beta=w\left(z_{0}, \mathbb{R} \backslash(a, b), \Pi_{+}\right)=\frac{\pi-\vartheta}{\pi}, \quad \vartheta=\arg \frac{z-b}{z-a},
$$

где $\vartheta$ - угол при вершине $z_{0}$ треугольника с вершинами в точках $a, b$ и $z_{0}$. Для функции $h_{(a, b)}$ справедливо равенство

$$
h_{(a, b)}(z)=\left(\frac{z-a}{z-b}\right)^{i / \pi}=\exp \left\{-\frac{i}{\pi} \ln \left(\frac{z-b}{z-a}\right)\right\} .
$$

Экстремальная функция (1.8) в теореме 1 имеет вид

$$
F(z)=\left(\frac{y_{0}}{\pi-\vartheta}\right)^{1 / p} \frac{1}{\left(z-\overline{z_{0}}\right)^{2 / p}}\left(\frac{z-a}{z-b}\right)^{i / \pi\left(\ln \delta+\frac{1}{p} \ln \frac{\pi-\vartheta}{\vartheta}\right)} g_{\phi}^{1 / p}(z), \quad z_{0}=x_{0}+i y_{0}
$$

Функционал (1.11) оптимального восстановления (наилучшего приближения) $T_{\delta}$ для случая $\mathbb{I}=(a, b)$ примет вид

$$
T_{\delta} f=\mathcal{K} \delta^{\vartheta / \pi-1} \int_{a}^{b}\left[\frac{1}{\pi} \frac{y_{0}}{\left(x-x_{0}\right)^{2}+y_{0}^{2}}\right]^{1-1 / p} \phi^{1 / p}(x) \rightsquigarrow(x) f(x) d x,
$$

где функция æ( $x)=æ\left(z_{0}, p, \phi ; x\right)$ с модулем, тождественно равным единице, задается равенством

$$
æ(x):=\exp i\left\{\frac{2}{p} \operatorname{arctg} \frac{y_{0}}{x-x_{0}}-\frac{\pi}{p}+\frac{1}{\pi}\left(\ln \delta+\frac{1}{p} \ln \frac{\pi-\vartheta}{\vartheta}\right) \ln \frac{\left|z_{0}-a\right|(b-x)}{\left|z_{0}-b\right|(x-a)}-\frac{1}{p} \arg \left(g_{\phi}(x)\right)\right\},
$$

и величина $\mathcal{K}-$ равенством

$$
\mathcal{K}:=\left\{\frac{g_{\phi}\left(z_{0}\right)}{4 \pi y_{0}}\left(\frac{\vartheta}{\pi-\vartheta}\right)^{1-\vartheta / \pi}\right\}^{1 / p} .
$$

Соответственно, точное неравенство (1.2) для функций класса $\mathcal{H}=\mathcal{H}^{p}\left(\Pi_{+}, \phi\right)$ примет вид

$$
\left|f\left(z_{0}\right)\right| \leq \mathcal{C}\|f\|_{L_{\phi}^{p}(a, b)}^{\vartheta / \pi}\|f\|_{L_{\phi}^{p}(\mathbb{R} \backslash(a, b))}^{1-\vartheta / \pi}, \quad f \in \mathcal{H}^{p}\left(\Pi_{+}, \phi\right),
$$

величина $\mathcal{C}$ задается равенством

$$
\mathcal{C}=\left\{\frac{\left|g_{\phi}\left(z_{0}\right)\right|}{4 y_{0}(\pi-\vartheta)}\left(\frac{\vartheta}{\pi-\vartheta}\right)^{-\vartheta / \pi}\right\}^{1 / p}
$$

\section{2. Экстремальные функция и функционал в случае $\mathbb{I}=(0,+\infty)$}

В случае, когда промежуток $\mathbb{I}$ является полупрямой $\mathbb{R}_{+}=(0,+\infty)$, для гармонических мер имеем

$$
\alpha=w\left(z_{0},(0,+\infty), \Pi_{+}\right)=\frac{\pi-\varphi_{0}}{\pi}, \quad \beta=w\left(z_{0},(-\infty, 0), \Pi_{+}\right)=\frac{\varphi_{0}}{\pi}, \quad \varphi_{0}=\arg z_{0} .
$$

Для функции $h_{\mathbb{R}_{+}}$справедливо равенство

$$
h_{\mathbb{R}_{+}}(z):=e z^{i / \pi}=\exp \left\{1+\frac{i}{\pi} \ln z\right\}
$$


Экстремальная функция (1.8) в теореме 1 имеет вид

$$
F(z)=\left(\frac{y_{0}}{\pi-\varphi_{0}}\right)^{1 / p} \frac{\delta}{\left(z-\overline{z_{0}}\right)^{2 / p}} z^{i / \pi\left(\ln \delta+\frac{1}{p} \ln \frac{\varphi_{0}}{\pi-\varphi_{0}}\right)} g_{\phi}^{1 / p}(z), \quad z_{0}=\left|z_{0}\right| e^{i \varphi_{0}}=x_{0}+i y_{0} .
$$

Заметим, что в случае $p=\infty$ экстремальная функция $F(z)=\delta z^{i \ln \delta / \pi}$ не зависит от точки $z_{0}$.

Функционал (1.11) оптимального восстановления (наилучшего приближения) $T_{\delta}$ для полуоси примет вид

$$
T_{\delta} f=\mathcal{K} \delta^{-\varphi_{0} / \pi} \int_{0}^{+\infty}\left[\frac{1}{\pi} \frac{y_{0}}{\left(x-x_{0}\right)^{2}+y_{0}^{2}}\right]^{1-1 / p} \phi^{1 / p}(x) \rightsquigarrow(x) f(x) d x,
$$

где функция $æ(x)=æ\left(z_{0}, p, \phi ; x\right)$ с модулем, тождественно равным единице, задается равенством

$$
æ(x):=\exp i\left\{\frac{2}{p} \operatorname{arctg} \frac{y_{0}}{x-x_{0}}-\frac{\pi}{p}+\frac{1}{\pi}\left(\ln \delta+\frac{1}{p} \ln \frac{\varphi_{0}}{\pi-\varphi_{0}}\right) \ln \frac{\left|z_{0}\right|}{x}-\frac{1}{p} \arg \left(g_{\phi}(x)\right)\right\},
$$

и величина $\mathcal{K}-$ равенством

$$
\mathcal{K}:=\left\{\frac{g_{\phi}\left(z_{0}\right)}{4 \pi y_{0}}\left(\frac{\pi-\varphi_{0}}{\varphi_{0}}\right)^{\varphi_{0} / \pi}\right\}^{1 / p}
$$

Соответственно точное неравенство (1.2) для функций класса $\mathcal{H}=\mathcal{H}^{p}\left(\Pi_{+}, \phi\right)$ примет вид

$$
\left|f\left(z_{0}\right)\right| \leq \mathcal{C}\|f\|_{L_{\phi}^{p}(-\infty, 0)}^{\varphi_{0} / \pi}\|f\|_{L_{\phi}^{p}(0,+\infty)}^{1-\varphi_{0} / \pi}, \quad f \in \mathcal{H}^{p}\left(\Pi_{+}, \phi\right),
$$

величина $\mathcal{C}$ задается равенством

$$
\mathcal{C}=\left\{\frac{\left|g_{\phi}\left(z_{0}\right)\right|}{4 \varphi_{0} y_{0}}\left(\frac{\pi-\varphi_{0}}{\varphi_{0}}\right)^{\varphi_{0} / \pi-1}\right\}^{1 / p} .
$$

\section{2. Оптимальное восстановление на луче аналитической в полуплоскости функции по приближенно заданным граничным значениям на полуоси}

В этой части статьи будем использовать результаты разд. 1 в случае, когда промежутком $\mathbb{I}$ является положительная полуось $\mathbb{R}_{+}$при $p=\infty$. В этом случае вид экстремальные функция и функционал в теореме 1 наиболее простой. А именно, справедливы равенства

$$
\begin{gathered}
F(z)=F\left[\mathbb{R}_{+}, \infty, \delta\right](z)=\delta z^{i / \pi \ln \delta}, \\
T_{\delta} f=T\left[z_{0}, \mathbb{R}_{+}, \infty, \delta\right] f=\delta^{-\varphi_{0} / \pi} \int_{0}^{+\infty}\left[\frac{1}{\pi} \frac{y_{0}}{\left(x-x_{0}\right)^{2}+y_{0}^{2}}\right] \rightsquigarrow(x) f(x) d x,
\end{gathered}
$$

где $æ(x)=\exp \left\{i / \pi \ln \delta \ln \frac{\left|z_{0}\right|}{x}\right\}$.

Точное неравенство $(1.2)$ в этом случае (с константой $\mathcal{C}=1)$ принимает вид

$$
\left|f\left(r e^{i \varphi_{0}}\right)\right| \leq\|f\|_{L^{\infty}(-\infty, 0)}^{\varphi_{0} / \pi}\|f\|_{L^{\infty}(0,+\infty)}^{1-\varphi_{0} / \pi}, \quad 0<r<\infty .
$$

Через $\gamma_{\alpha}$ обозначим луч

$$
\gamma_{\alpha}:=\left\{z \in \mathbb{C}: \arg z=\varphi_{0}\right\}=e^{i \varphi_{0}} \mathbb{R}_{+} .
$$


Будем использовать независимость экстремальной функции $F\left[\mathbb{R}_{+}, \infty, \delta\right]$ от точки $z_{0}$, постоянство гармонической меры на луче $\gamma_{\alpha}: w\left(z_{0}, \mathbb{R}_{+}, \Pi_{+}\right)=\alpha=1-\varphi_{0} / \pi, z_{0} \in \gamma_{\alpha}$, и однородность степени -1 ядра функционала (2.2). Рассмотрим задачи оптимального восстановления и наилучшего приближения оператора $\Upsilon_{\gamma_{\alpha}}$, сопоставляющего предельным граничным значениям функции $f \in \mathcal{H}$ на положительной полуоси $\mathbb{R}_{+}$сужение этой функции на луче $\gamma_{\alpha}$.

Обозначим через $B$ банахово пространство функций, определенных на полупрямой $\gamma_{\alpha}$. Функцию переменной $\delta>0$, задаваемую равенством

$$
\omega(\delta):=\omega\left(\delta ; \Upsilon_{\gamma_{\alpha}}, Q\right)=\sup \left\{\|f\|_{B}: f \in Q,\|f\|_{L_{\phi}^{p}\left(\mathbb{R}_{+}\right)} \leq \delta\right\},
$$

называют модулем непрерывности оператора $\Upsilon_{\gamma_{\alpha}}$ на классе $Q$.

В качестве множества методов восстановления $\mathcal{R}$, из которых выбирается оптимальный, будем рассматривать множество $\mathcal{O}$ всех возможных, множество $\mathcal{L}$ линейных или множество $\mathcal{B}$ линейных ограниченных операторов из $L_{\phi}^{p}\left(\mathbb{R}_{+}\right)$в $B$. Для числа $\delta>0$ и метода восстановления $T \in \mathcal{R}$ величина

$$
\mathcal{U}(T, \delta):=\sup \left\{\left\|f\left(z_{0}\right)-T \widetilde{f}\right\|_{B}: f \in Q, \widetilde{f} \in L_{\phi}^{p}\left(\mathbb{R}_{+}\right),\|f-\widetilde{f}\|_{L_{\phi}^{p}\left(\mathbb{R}_{+}\right)} \leq \delta\right\}
$$

является погрешностью восстановления на луче $\gamma_{\alpha}$ функций класса $Q$ по их граничным значениям на $\mathbb{R}_{+}$, заданным с ошибкой $\delta$ по норме $L_{\phi}^{p}\left(\mathbb{R}_{+}\right)$, методом $T$. Тогда

$$
\mathcal{E}_{\mathcal{R}}(\delta):=\inf \{\mathcal{U}(T, \delta): T \in \mathcal{R}\}
$$

есть величина оптимального восстановления на луче $\gamma_{\alpha}$ (или, что то же самое, оптимального восстановления оператора $\Upsilon_{\gamma_{\alpha}}$ ) функций класса $Q$ по их $\delta$-приближенным граничным значениям на $\mathbb{R}_{+}$с помощью методов восстановления $\mathcal{R}$.

Постановка задачи наилучшего приближения оператора $\Upsilon_{\gamma_{\alpha}}$ линейными ограниченными операторами такова. Пусть $\mathcal{L}(N)$ есть множество линейных ограниченных операторов из $L_{\phi}^{p}\left(\mathbb{R}_{+}\right)$в $B$, норма которых не превосходит числа $N>0$. Величина

$$
U(T):=\sup \left\{\left\|f\left(z_{0}\right)-T f\right\|_{B}: f \in Q\right\}
$$

является уклонением оператора $T \in \mathcal{L}(N)$ от оператора $\Upsilon_{\gamma_{\alpha}}$ на классе функций $Q$. Соответственно величина

$$
E(N):=\inf \{U(T): T \in \mathcal{L}(N)\}
$$

есть наилучшее приближение оператора $\Upsilon_{\gamma_{\alpha}}$ множеством линейных ограниченных операторов $\mathcal{L}(N)$ на классе $Q$.

Решение задач (2.5) и (2.7) оптимального восстановления и наилучшего приближения оператора $\Upsilon_{\gamma_{\alpha}}$ получено в двух случаях.

(I) Классом $Q$ является класс $Q^{\infty}\left(\Pi_{+}, \mathbb{R}_{+}\right)$при $p=\infty$; пространство $B$ есть произвольная банахова решетка функций, определенных на полупрямой $\gamma_{\alpha}$, содержащая константы, с нормой, удовлетворяющей условию $\|1\|_{B}=1$.

(II) Классом $Q$ является класс $Q^{p}\left(\Pi_{+}, \mathbb{R}_{+}, \psi\right)$; пространство $B$ есть $L_{\psi}^{p}\left(\gamma_{\alpha}\right)$ с параметром $p, 1 \leq p<\infty$, и весом $\psi(z)=\psi_{1 / 2}(0, z)=1 /|z|$.

Рассмотрим оператор $\mathcal{T}_{\delta}$, определяемый с помощью функционала (2.2) равенством $\left(\mathcal{T}_{\delta} f\right)(z)=T\left[z, \mathbb{R}_{+}, \infty, \delta\right] f, z \in \gamma_{\alpha}$. Явный вид оператора задает равенство

$$
\begin{gathered}
\left(\mathcal{T}_{\delta} f\right)\left(r e^{i \varphi_{0}}\right):=\delta^{-\varphi_{0} / \pi} \frac{1}{\pi} \int_{0}^{+\infty} \frac{r \sin \varphi_{0}}{\left(r \cos \varphi_{0}-x\right)^{2}+r^{2} \sin ^{2} \varphi_{0}}\left(\frac{r}{x}\right)^{i / \pi \ln \delta} f(x) d x \\
=\delta^{-\varphi_{0} / \pi} \frac{1}{\pi} \int_{0}^{+\infty} \frac{\sin \varphi_{0}}{\left(\cos \varphi_{0}-t\right)^{2}+\sin ^{2} \varphi_{0}} t^{-i / \pi \ln \delta} f(r t) d t
\end{gathered}
$$


Теорема 2. В случаях (I) и (II) справедливы следующие утверждения.

1) Для величин (2.3) и (2.5) имеют место равенства

$$
\omega(\delta)=\mathcal{E}_{\mathcal{O}}(\delta)=\mathcal{E}_{\mathcal{B}}(\delta)=\mathcal{E}_{\mathcal{L}}(\delta)=\delta^{1-\varphi_{0} / \pi}
$$

Экстремальными в (2.3) являются функиии вида $\epsilon F,|\epsilon|=1$; в задаче (2.5) оптимальным методом восстановления является линейный ограниченный оператор $\mathcal{T}_{\delta}$.

2) Для функиий класса $\mathcal{H}^{\infty}\left(\Pi_{+}\right)$справедливо точное неравенство

$$
\|f\|_{B} \leq\|f\|_{L^{\infty}(-\infty, 0)}^{\varphi_{0} / \pi}\|f\|_{L^{\infty}(0,+\infty)}^{1-\varphi_{0} / \pi}
$$

Для функций класса $\mathcal{H}^{p}\left(\Pi_{+}, \psi\right), 1 \leq p<\infty, \psi(z)=1 /|z|$, справедливо точное неравенство

$$
\|f\|_{L_{\psi}^{p}\left(\gamma_{\alpha}\right)} \leq\|f\|_{L_{\psi}^{p}(-\infty, 0)}^{\varphi_{0} / \pi}\|f\|_{L_{\psi}^{p}(0,+\infty)}^{1-\varphi_{0} / \pi} .
$$

3) Для величины (2.7) справедливо равенство

$$
E(N)=\frac{\varphi_{0}}{\pi}\left(\frac{\pi-\varphi_{0}}{\pi}\right)^{\pi / \varphi_{0}-1} N^{1-\pi / \varphi_{0}} .
$$

В задаче (2.7) оператором наилучшего приближения является $\mathcal{T}_{\delta}$ с параметром

$$
\delta=\left(\frac{\pi-\varphi_{0}}{\pi}\right)^{\pi / \varphi_{0}} N^{-\pi / \varphi_{0}} .
$$

З а м е ч а н и е. Сравним случай (II) с работой [2], где рассматривались аналогичные (2.5) и (2.7) задачи для класса функций, аналитических в полосе. При отображении $\zeta=\ln z$ класс, рассмотренный в [2], переходит в класс $\mathfrak{H}^{p}\left(\Pi_{+}, \psi\right)$ функций, аналитических в полуплоскости, со следующим определением: это класс функций, которые имеют почти всюду на $\mathbb{R}$ некасательные предельные значения и сужение которых на произвольный луч $\gamma_{\alpha}, 0<\alpha<1$, принадлежит $L_{\psi}^{p}\left(\gamma_{\alpha}\right)$, при этом $\sup \left\{\|f\|_{L_{\psi}^{p}\left(\gamma_{\alpha}\right)}: 0<\alpha<1\right\}<+\infty$. Из [2, лемма 2] следует вложение $\mathfrak{H}^{p}\left(\Pi_{+}, \psi\right) \subset \mathcal{H}^{p}\left(\Pi_{+}, \psi\right)$. Обратное вложение $\mathcal{H}^{p}\left(\Pi_{+}, \psi\right) \subset \mathfrak{H}^{p}\left(\Pi_{+}, \psi\right)$ является следствием теоремы 2, а точнее неравенства (2.12). Таким образом, эти классы совпадают и, следовательно, задачи (2.5), (2.7) совпадают с задачами, рассмотренными в [2] с точностью до отображения $\zeta=\ln z$.

\section{3. Вспомогательные утверждения}

Лемма 1. Справедливы утверждения:

1. Для модуля непрерывности (1.1) функиионала $\Upsilon_{z_{0}}$ на классе $Q^{p}\left(\Pi_{+}, \mathbb{I}, \phi\right)$ имеет место неравенство $\omega(\delta) \geq \mathcal{C} \delta^{\alpha}$.

2. В случалх (I) и (II) для модуля непрерывности (2.3) оператора $\Upsilon_{\gamma_{\alpha}}$ имеет место неравенство $\omega(\delta) \geq \delta^{\alpha}$.

Д о к а з а т е л ь с т в о. Для оценки снизу модуля непрерывности (1.1) функционала $\Upsilon_{z_{0}}$ используем функцию $F=F\left[z_{0}, \mathbb{I}, p, \phi, \delta\right]$ из класса $Q=Q^{p}\left(\Pi_{+}, \mathbb{I}, \phi\right)$, определенную в $(1.8)$, и равенство (1.10). Имеем $\omega\left(\delta ; \Upsilon_{z_{0}}, Q\right) \geq\left|F\left(z_{0}\right)\right|=\mathcal{C} \delta^{\alpha}$.

В случае $(\mathbf{I})$ для оценки модуля непрерывности (2.3) оператора $\Upsilon_{\gamma_{\alpha}}$ используем функцию $F=F\left[\mathbb{R}_{+}, \infty, \delta\right]$ из класса $Q=Q^{\infty}\left(\Pi_{+}, \mathbb{R}_{+}\right)$, определенную в (2.1). Получим неравенство

$$
\omega\left(\delta ; \Upsilon_{\gamma_{\alpha}}, Q\right) \geq\left|F\left(z_{0}\right)\right|=\delta^{\alpha} .
$$


В случае (II) рассмотрим последовательность $F_{n}(z):=F\left[\mathbb{R}_{+}, \infty, \delta\right](z) z^{1 / n}(z+i)^{-2 / n}$. Обозначим через $I_{n}(\varphi)$ величину, определяемую равенством

$$
I_{n}(\varphi):=\int_{0}^{+\infty}\left(\frac{r}{\left|r e^{i \varphi}+i\right|^{2}}\right)^{p / n} \frac{d r}{r}=\int_{0}^{+\infty} r^{p / n-1}\left(r^{2}+2 r \sin \varphi+1\right)^{-p / n} d r .
$$

Нетрудно понять, что наименьшее и наибольшее значения $I_{n}(\varphi)$ на отрезке $[0, \pi]$ достигаются, соответственно, в точках $\varphi=\pi / 2$ и $\varphi=0(\varphi=\pi)$. Вычислив интегралы $I_{n}(\varphi)$ для экстремальных точек $\varphi$, получим, что для произвольного $\varphi, 0 \leq \varphi \leq \pi$, справедливы соотношения

$$
B\left(\frac{p}{n}, \frac{p}{n}\right)=I_{n}\left(\frac{\pi}{2}\right) \leq I_{n}(\varphi) \leq I_{n}(0)=I_{n}(\pi)=\frac{1}{2} B\left(\frac{p}{2 n}, \frac{p}{2 n}\right),
$$

в котором $B$ есть $B$-функция Эйлера. Отсюда получим неравенство

$$
\frac{2 B\left(\frac{p}{n}, \frac{p}{n}\right)}{B\left(\frac{p}{2 n}, \frac{p}{2 n}\right)}=\frac{I_{n}\left(\frac{\pi}{2}\right)}{I_{n}(\pi)} \leq \frac{I_{n}(\varphi)}{I_{n}(\pi)} \leq 1 .
$$

Замечая, что предел последовательности в левой части последнего неравенства равен единице, получим

$$
\lim _{n \rightarrow \infty} \frac{I_{n}(\varphi)}{I_{n}(\pi)}=1, \quad 0 \leq \varphi \leq \pi .
$$

Нормы функции $F_{n}$ и интегралы $I_{n}$ связаны равенствами

$$
\left\|F_{n}\right\|_{L_{\psi}^{p}(-\infty, 0)}^{p}=I_{n}(\pi), \quad\left\|F_{n}\right\|_{L_{\psi}^{p}(0,+\infty)}^{p}=\delta^{p} I_{n}(0), \quad\left\|F_{n}\right\|_{L_{\psi}^{p}\left(\gamma_{\alpha}\right)}^{p}=\delta^{p \alpha} I_{n}\left(\varphi_{0}\right) .
$$

Тогда для произвольного $n \in \mathbb{N}$ функция $I_{n}^{-1 / p}(\pi) F_{n}$ принадлежит классу $Q=Q^{p}\left(\Pi_{+}, \mathbb{I}, \psi\right)$, при этом $\left\|I_{n}^{-1 / p}(\pi) F_{n}\right\|_{L_{\psi}^{p}(0,+\infty)}=\delta$. Теперь, используя соотношение (3.1), получим оценку модуля непрерывности $\omega\left(\delta ; \Upsilon_{\gamma_{\alpha}}, Q\right) \geq \lim _{n \rightarrow \infty}\left\|I_{n}^{-1 / p}(\pi) F_{n}\right\|_{L_{\psi}^{p}\left(\gamma_{\alpha}\right)}=\delta^{\alpha} \lim _{n \rightarrow \infty} \frac{I_{n}^{1 / p}\left(\varphi_{0}\right)}{I_{n}^{1 / p}(\pi)}=\delta^{\alpha}$. Лемма 1 доказана.

Лемма 2. Справедливы утверждения:

1. Для нормы функционала $T_{\delta}$ на пространстве $L_{\phi}^{p}(\mathbb{I})$, уклонений (1.3) и (1.5) функционала $T_{\delta}$ от функиионала $\Upsilon_{z_{0}}$ на классе функиий $Q^{p}\left(\Pi_{+}, \mathbb{I}, \phi\right)$ имеют место равенства

$$
\left\|T_{\delta}\right\|=\alpha \mathcal{C} \delta^{-\beta}, \quad U\left(T_{\delta}\right)=\beta \mathcal{C} \delta^{\alpha}, \quad \mathcal{U}\left(T_{\delta}, \delta\right)=\mathcal{C} \delta^{\alpha} .
$$

2. В случаях (I) и (II) для нормы оператора $\mathcal{T}_{\delta}$, уклонений $(2.4)$ и (2.6) оператора $\mathcal{T}_{\delta}$ от оператора $\Upsilon_{\gamma_{\alpha}}$ именот место равенства

$$
\left\|\mathcal{T}_{\delta}\right\|=\alpha \delta^{-\beta}, \quad U\left(\mathcal{T}_{\delta}\right)=\beta \delta^{\alpha}, \quad \mathcal{U}\left(\mathcal{T}_{\delta}, \delta\right)=\delta^{\alpha} .
$$

Д о к а з а т е л ь с т в о. Из определения (1.11) функционала $T_{\delta}$, равенств (1.9) и (1.10) имеем

$$
\left|T_{\delta} f\right| \leq C \delta^{\alpha} \int_{\mathbb{I}} P^{1-1 / p}\left(x, z_{0}\right) \delta^{-1} \alpha^{1 / p}|f(x)| \phi^{1 / p}(x) d x \leq \alpha C \delta^{-\beta}\|f\|_{L_{\phi}^{p}(\mathbb{I})} .
$$

Неравенство обращается в равенство на функции $F=F\left[z_{0}, \mathbb{I}, p, \phi, \delta\right]$, определенной в (1.8). Тем самым вычислена норма функционала $T_{\delta}$.

Для оценок уклонений вначале покажем, что для произвольной функции $f \in Q$ функция $f / F$, где $F=F\left[z_{0}, \mathbb{I}, p, \phi, \delta\right]$ определена равенством (1.8), принадлежит классу Хар- 
ди $H^{1}\left(\Pi_{+}\right)$. Из определений класса $Q$ и функции $F$ следует, что $f$ и $1 / F$ из класса $N_{*}$, тогда и $f / F \in N_{*}$. Из равенств (1.9) получим оценку

$$
\begin{gathered}
\int_{-\infty}^{+\infty} \frac{|f(x)|}{F(x)} P\left(x, z_{0}\right) d x \leq \mu \int_{-\infty}^{+\infty} P^{1-1 / p}\left(x, z_{0}\right)|f(x)| \phi^{1 / p}(x) d x \\
\leq \mu\left(\int_{-\infty}^{+\infty} P\left(x, z_{0}\right) d x\right)^{1-1 / p}\|f\|_{L_{\phi}^{p}(\mathbb{R})}=\mu\|f\|_{L_{\phi}^{p}(\mathbb{R})},
\end{gathered}
$$

где $\mu=\max \left\{\delta^{-1} \alpha^{1 / p}, \beta^{1 / p}\right\}$. Итак, функция $f / F$ принадлежит классу $N_{*}$ и суммируема по гармонической мере на оси, тогда $f / F \in H^{1}\left(\Pi_{+}\right)$.

Из принадлежности функции $f / F$ классу Харди $H^{1}\left(\Pi_{+}\right)$следует представимость этой функции по формуле Грина через ее предельные граничные значения. Отсюда получаем равенство

$$
f\left(z_{0}\right)-T_{\delta} f=\int_{-\infty}^{0} P\left(x, z_{0}\right) \frac{F\left(z_{0}\right)}{F(x)} f(x) d x
$$

Тогда для произвольной функции $f \in Q$ верна оценка

$$
\left|f\left(z_{0}\right)-T_{\delta} f\right| \leq \beta^{1 / p}\left|F\left(z_{0}\right)\right| \int_{-\infty}^{0} P^{1-1 / p}\left(x, z_{0}\right) f(x) \phi^{1 / p}(x) d x \leq \beta\left|F\left(z_{0}\right)\right|\|f\|_{L_{\phi}^{p}(\mathbb{R})}=\beta \mathcal{C} \delta^{\alpha} .
$$

Последнее неравенство обращается в равенство на функции $F=F\left[z_{0}, \mathbb{I}, p, \phi, \delta\right]$. Получили $U\left(T_{\delta}\right)=\beta \mathcal{C} \delta^{\alpha}$.

Для уклонения (1.3) справедлива оценка сверху

$$
\mathcal{U}\left(T_{\delta}, \delta\right) \leq U\left(T_{\delta}\right)+\left\|T_{\delta}\right\| \delta=\beta \mathcal{C} \delta^{\alpha}+\alpha C \delta^{-\beta} \delta=\mathcal{C} \delta^{\alpha} .
$$

Оценку снизу уклонения (1.3) дает пара функций $f=F\left[z_{0}, \mathbb{I}, p, \phi, \delta\right] \in Q$ и $\widetilde{f} \equiv 0$. Получим равенство $\mathcal{U}\left(T_{\delta}, \delta\right)=\mathcal{C} \delta^{\alpha}$.

Первое утверждение леммы доказано.

Рассмотрим случай $(\mathbf{I})$, т. е. исследуем норму оператора $\mathcal{T}_{\delta}$, определенного формулой $(2.8)$, из $L^{\infty}\left(\mathbb{R}_{+}\right)$в $B$, и его уклонения на классе $Q^{\infty}\left(\Pi_{+}, \mathbb{R}_{+}\right)$по норме банаховой решетки $B$. В случае $p=\infty$ экстремальная функция $F=F\left[\mathbb{R}_{+}, \infty, \delta\right]=\delta z^{i / \pi \ln \delta}$ и величина $\mathcal{C}=1$ не зависят от точки $z_{0}$, а значения гармонических мер $\alpha=1-\varphi_{0} / \pi, \beta=\varphi_{0} / \pi$ и модуль функции $F$ постоянны на луче $\gamma_{\alpha}$. Тогда, используя определение оператора, свойства нормы пространства $B$ и первое утверждение леммы, получаем

$$
\left\|\mathcal{T}_{\delta}\right\|=\left\|\alpha \mathcal{C} \delta^{-\beta}\right\|_{B}=\alpha \delta^{-\beta}, \quad U\left(\mathcal{T}_{\delta}\right)=\left\|\beta \mathcal{C} \delta^{\alpha}\right\|_{B}=\beta \delta^{\alpha}, \quad \mathcal{U}\left(\mathcal{T}_{\delta}, \delta\right)=\left\|\mathcal{C} \delta^{\alpha}\right\|_{B}=\delta^{\alpha} .
$$

Рассмотрим случай $(\mathbf{I I})$, соответственно, исследуем норму из $L_{\psi}^{p}\left(\mathbb{R}_{+}\right)$в $L_{\psi}^{p}\left(\gamma_{\alpha}\right)$ и уклонения на классе $Q^{p}\left(\Pi_{+}, \mathbb{R}_{+}, \psi\right)$ по норме $L_{\psi}^{p}\left(\gamma_{\alpha}\right)$ для оператора $\mathcal{T}_{\delta}$, определенного в $(2.8)$. Используем представление (2.9), получим оценку

$$
\begin{gathered}
\left\|\mathcal{T}_{\delta} f\right\|_{L_{\psi}^{p}\left(\gamma_{\alpha}\right)} \leq \delta^{-\varphi_{0} / \pi} \frac{1}{\pi} \int_{0}^{+\infty} \frac{\sin \varphi_{0} d t}{\left(\cos \varphi_{0}-t\right)^{2}+\sin ^{2} \varphi_{0}}\left(\int_{0}^{+\infty}|f(r t)|^{p} \frac{d r}{r}\right)^{1 / p} \\
=\delta^{-\varphi_{0} / \pi} w\left(e^{i \varphi_{0}}, \mathbb{R}_{+}, \Pi_{+}\right)\|f\|_{L_{\psi}^{p}\left(\mathbb{R}_{+}\right)}=\alpha \delta^{-\beta}\|f\|_{L_{\psi}^{p}\left(\mathbb{R}_{+}\right)} .
\end{gathered}
$$

Тогда для нормы оператора $\mathcal{T}_{\delta}$ имеет место оценка сверху

$$
\left\|\mathcal{T}_{\delta}\right\|_{L_{\psi}^{p}\left(\mathbb{R}_{+}\right) \rightarrow L_{\psi}^{p}\left(\gamma_{\alpha}\right)} \leq \alpha \delta^{-\beta}
$$


Формула (3.2) влечет равенство

$$
f\left(r e^{i \varphi_{0}}\right)-\left(\mathcal{T}_{\delta} f\right)\left(r e^{i \varphi_{0}}\right)=\delta^{-\varphi_{0} / \pi} \frac{1}{\pi} \int_{-\infty}^{0} \frac{\sin \varphi_{0}}{\left(\cos \varphi_{0}-t\right)^{2}+\sin ^{2} \varphi_{0}} t^{-i \ln \delta / \pi} f(r t) d t .
$$

Следовательно, справедливо

$$
\left\|f-\mathcal{T}_{\delta} f\right\|_{L_{\psi}^{p}\left(\gamma_{\alpha}\right)}=\delta^{1-\varphi_{0} / \pi} \frac{1}{\pi} \int_{-\infty}^{0} \frac{\sin \varphi_{0} d t}{\left(\cos \varphi_{0}-t\right)^{2}+\sin ^{2} \varphi_{0}}\left(\int_{0}^{+\infty}|f(r t)|^{p} \frac{d r}{r}\right)^{1 / p}=\beta \delta^{\alpha}\|f\|_{L_{\psi}^{p}\left(\mathbb{R}_{-}\right)} .
$$

Тогда для уклонения (2.6) оператора $\mathcal{T}_{\delta}$ имеет место оценка сверху

$$
U\left(\mathcal{T}_{\delta}\right) \leq \beta \delta^{\alpha}
$$

Наконец, для уклонения (2.4) справедливо неравенство

$$
\mathcal{U}\left(\mathcal{T}_{\delta}, \delta\right) \leq U\left(\mathcal{T}_{\delta}\right)+\left\|\mathcal{T}_{\delta}\right\| \delta \leq \delta^{\alpha}
$$

Покажем, что справедливо неравенство $\mathcal{U}\left(\mathcal{T}_{\delta}, \delta\right) \geq \delta^{\alpha}$, откуда получим, что в $(3.5)$, а следовательно в (3.3) и (3.4), неравенства являются равенствами. В определении (2.4) уклонения положим $f=I_{n}^{-1 / p}(\pi) F_{n}, F_{n}(z)=F(z) z^{1 / n}(z+i)^{-2 / n}$ и $\widetilde{f} \equiv 0$. Получим неравенство

$$
\mathcal{U}\left(\mathcal{T}_{\delta}, \delta\right) \geq \lim _{n \rightarrow \infty}\left\|I_{n}^{-1 / p}(\pi) F_{n}-\mathcal{T}_{\delta} 0\right\|_{L_{\psi}^{p}\left(\gamma_{\alpha}\right)}=\lim _{n \rightarrow \infty}\left\|I_{n}^{-1 / p}(\pi) F_{n}\right\|_{L_{\psi}^{p}\left(\gamma_{\alpha}\right)}=\delta^{\alpha} .
$$

Лемма 2 доказана.

\section{4. Доказательства теорем}

Для доказательства теорем 1 и 2 будем использовать стандартную схему. Известна взаимосвязь задачи Стечкина о наилучшем приближении неограниченного оператора линейными ограниченными операторами с задачами оптимального восстановления и модулем непрерывности оператора. Для задач (1.4), (1.6) и модуля непрерывности (1.1) для функционала $\Upsilon_{z_{0}}$ и, соответственно, задач $(2.5),(2.7),(2.3)$ для оператора $\Upsilon_{\gamma_{\alpha}}$ эта взаимосвязь выражается следующим образом. Введем обозначения

$$
\begin{array}{cl}
\Delta(N):=\sup \{\omega(\delta)-N \delta: \delta \geq 0\}, & N>0, \\
l(\delta):=\inf \{E(N)+N \delta: N>0\}, & \delta \geq 0 .
\end{array}
$$

Как частный случай результата С. Б. Стечкина (см. [8, теорема 1.1]), справедливы неравенства

$$
\begin{gathered}
\Delta(N) \leq E(N), \quad N>0 . \\
\omega(\delta) \leq l(\delta) .
\end{gathered}
$$

Следующее уточнение неравенства (4.2) является частным случаем общего утверждения, связывающего задачу о модуле непрерывности оператора и задачу Стечкина с задачами оптимального восстановления (см. [8, теорема 2.1])

$$
\omega(\delta) \leq \mathcal{E}_{\mathcal{O}}(\delta) \leq \mathcal{E}_{\mathcal{L}}(\delta)=\mathcal{E}_{\mathcal{B}}(\delta) \leq l(\delta) .
$$

Для задач наилучшего приближения и оптимального восстановления функционала неравенства в соотношениях (4.1), (4.2) являются равенствами и соотношению (4.3) соответствует равенство $\omega(\delta)=\mathcal{E}(\delta)=l(\delta)$. 
Д о к а з а т е л ь с т в о теоремы 1. Для величин модуля непрерывности (1.1) и оптимального восстановления (1.4) функционала $\Upsilon_{z_{0}}$ на классе $Q^{p}\left(\Pi_{+}, \mathbb{I}, \phi\right)$, применяя леммы 1 и 2 , а также формулу (4.3), получим цепочку соотношений

$$
\mathcal{C} \delta^{\alpha} \leq \omega\left(\delta ; \Upsilon_{z_{0}}, Q\right)=\mathcal{E}(\delta) \leq \mathcal{U}\left(T_{\delta}, \delta\right)=\mathcal{C} \delta^{\alpha}
$$

Отсюда следует первое утверждение теоремы 1 . Теперь, зная $\omega(\delta)$, вычислим по определению величину $\Delta(N)$. Получим равенство $\Delta(N)=\mathcal{C}^{1 / \beta} \beta \alpha^{\alpha / \beta} N^{-\alpha / \beta}$. Для величины наилучшего приближения (1.6) функционала $\Upsilon_{z_{0}}$ на классе $Q^{p}\left(\Pi_{+}, \mathbb{I}, \phi\right)$, используя лемму 2 при $\delta=\mathcal{C}^{1 / \beta} \alpha^{1 / \beta} N^{-1 / \beta}$ и формулу (4.1), получим цепочку соотношений

$$
\mathcal{C}^{1 / \beta} \beta \alpha^{\alpha / \beta} N^{-\alpha / \beta}=\Delta(N)=E(N) \leq U\left(T_{\delta}\right)=\mathcal{C}^{1 / \beta} \beta \alpha^{\alpha / \beta} N^{-\alpha / \beta} .
$$

Отсюда следует второе утверждение теоремы 1.

Д о к а з а т е л ь с т в о теоремы 2 в обоих случаях (I) и (II) проводится аналогично. Точнее, следует из соотношений для величин модуля непрерывности (2.3), оптимального восстановления (2.5) и наилучшего приближения (2.7) оператора $\Upsilon_{\gamma_{\alpha}}$, получаемых из лемм 1 и 2 , а также неравенств (4.1) и (4.3),

$$
\begin{gathered}
\delta^{\alpha} \leq \omega\left(\delta ; \Upsilon_{\gamma_{\alpha}}, Q\right) \leq \mathcal{E}_{\mathcal{O}}(\delta) \leq \mathcal{E}_{\mathcal{L}}(\delta)=\mathcal{E}_{\mathcal{B}}(\delta) \leq \mathcal{U}\left(\mathcal{T}_{\delta}, \delta\right)=\delta^{\alpha} \\
\beta \alpha^{\alpha / \beta} N^{-\alpha / \beta}=\Delta(N)=E(N) \leq U\left(\mathcal{T}_{\delta}\right)=\beta \alpha^{\alpha / \beta} N^{-\alpha / \beta} .
\end{gathered}
$$

Неравенства (2.11) и (2.12) следуют из определения модуля непрерывности и равенства (2.10).

Теорема 2 доказана.

Автор выражает благодарность В.В.Арестову за постоянную поддержку и плодотворные обсуждения.

\section{СПИСОК ЛИТЕРАТУРЫ}

1. Айзенберг Л.А. Формулы Карлемана в комплексном анализе. Первые приложения. Новосибирск: Наука. 1990. 248 с.

2. Акопян P.P. Наилучшее приближение оператора аналитического продолжения на классе аналитических в полосе функций // Тр. ИММ УрО РАН. Т. 17,№ 3, 2011. С. 46-54.

3. Акопян P.P. Оптимальное восстановление аналитической функции по заданным с погрешностью граничным значениям // Мат. заметки. 2016. Т. 99, № 2. С. 163-170.

4. Акопян P.P. Наилучшее приближение функционала аналитического продолжения с части границы // Современные проблемы теории функций и их приложения: материалы 18-й междунар. Сарат. зимней шк. Саратов: ООО Издательство "Научная книга", 2016. С. 25-26.

5. Akopyan R.R. Optimal recovery of a derivative of an analytic function from values of the function given with an error on a part of the boundary // Analysis Math. 2018. Vol. 44, no. 1. P. 3-19. doi: 10.1007/s10476-018-0102-7.

6. Арестов В.В. О равномерной регуляризации задачи вычисления значений оператора // Мат. заметки. 1977. Т. 22, по. 2. С. 231-244.

7. Арестов В.В. Наилучшее восстановление операторов и родственные задачи // Тр. МИАН. 1989. T. 189 . C. $3-20$.

8. Арестов В.В. Приближение неограниченных операторов ограниченными и родственные экстремальные задачи // Успехи мат. наук. 1996. Т. 51, вып. 6 (312). С. 89-124.

9. Arestov V., Filatova M. Best approximation of the differentiation operator in the space $L_{2}$ on the semiaxis // J. Approx. Theory. 2014. Vol. 187, no. l. P. 65-81. doi: 10.1016/j.jat.2014.08.001.

10. Gonzalez-Vera P., Stessin M.I. Joint spectra of Toeplitz operators and optimal recovery of analytic functions // Constr. Approx. 2012. Vol. 36, no. 1. P. 53-82. doi: 10.1007/s00365-012-9169-8.

11. DeGraw A. Optimal recovery of holomorphic functions from inaccurate information about radial integration // Amer. J. Comput. Math. 2012. Vol. 2, no. 4. P. 258-268. doi: 10.4236/ajcm.2012.24035.

12. Лаврентьев М.М., Романов В.Г., Шишатский С.П. Некорректные задачи математической физики и анализа. М.: Наука, 1980. 286 с. 
13. Магарил-Ильяев Г.Г., Осипенко К.Ю. Об оптимальном восстановлении функционалов по неточным данным // Мат. заметки. 1991. Т. 50, № 6. С. 85-93.

14. Micchelli Ch.A., Rivlin Th.J. A survey of optimal recovery // Optimal estimation in approximation theory. N.Y. etc.: Plenum Press, 1977. P. 1-54. doi: 10.1007/978-1-4684-2388-4_1.

15. Магарил-Ильяев Г.Г., Тихомиров В.М., Осипенко К.Ю. Неопределенность знания об объекте и точность методов его восстановления / / Проблемы передачи информации. 2003. Т. 39, вып 1. C. $118-133$.

16. Osipenko K.Yu. Optimal recovery of analytic functions. Huntington: NJVA Science Publ. Inc., 2000. 229 p. ISBN: 1-56072-821-3.

17. Осипенко К.Ю. Неравенство Харди - Литтлвуда - Полиа для аналитических функций из пространств Харди - Соболева // Мат. сб. 2006. Т. 197, № 3. С. 15-34.

18. Osipenko K.Y., Stessin M.I. Hadamard and Schwarz type theorems and optimal recovery in spaces of analytic functions // Constr. Approx. 2010. Vol. 31. P. 37-67. doi: 10.1007/s00365-009-9043-5 .

19. Осипенко К.Ю. Оптимальное восстановление линейных операторов в неевклидовых метриках // Мат. сб. 2014. Т. 205, № 10. С. 77-106.

Поступила 12.08.2018

После доработки 14.11.2018

Принята к публикации 19.11.2018

Акопян Роман Размикович

канд. физ.-мат. наук, доцент

Уральский федеральный университет;

Институт математики и механики им. Н. Н. Красовского УрО РАН,

г. Екатеринбург

e-mail: RRAkopyan@mephi.ru

\section{REFERENCES}

1. Ajzenberg L.A. Carleman formulas in complex analysis. First applications. Novosibirsk: Nauka, 1990, 248 p. (in Russian).

2. Akopyan R.R, Best approximation of the operator of analytic continuation on the class of functions analytic in a strip. Trudy Inst. Mat. i Mekh. UrO RAN, 2011, vol. 17, no. 3, pp. 46-54 (in Russian).

3. Akopyan R.R. Optimal recovery of analytic functions from boundary conditions specified with error. Math. Notes, 2016, vol. 99, no. 2, pp. 177-182. doi: 10.1134/S000143461601020X.

4. Akopyan R.R. Best approximation of the functional of the analytic continuation from a part of the boundary. Proc. 18th Int. Saratov Winter School "Contemporary Problems of Function Theory and Their Applications". Saratov: Nauchnaya kniga Publ., 2016, pp. 25-26 (in Russian). ISBN: 978-5-9758-1623-8.

5. Akopyan R.R. Optimal recovery of a derivative of an analytic function from values of the function given with an error on a part of the boundary. Analysis Math., 2018, vol. 44, no. 1, pp. 3-19. doi: 10.1007/s10476-018-0102-7.

6. Arestov V.V. Uniform regularization of the problem of calculating the values of an operator. Math. Notes, 1977, vol. 22, no. 2, pp. 618-626. doi: 10.1007/BF01780971.

7. Arestov V.V. Optimal recovery of operators and related problems. Proc. Steklov Inst. Math., 1990, no. 4, pp. 1-20.

8. Arestov V.V. Approximation of unbounded operators by bounded operators and related extremal problems. Russian Math. Surveys, 1996, vol. 51, no. 6, pp. 1093-1126. doi: 10.1070/RM1996v051n06ABEH003001.

9. Arestov V., Filatova M. Best approximation of the differentiation operator in the space $L_{2}$ on the semiaxis. J. Approx. Theory, 2014, vol. 187, no. 1, pp. 65-81. doi: 10.1016/j.jat.2014.08.001.

10. Gonzalez-Vera P., Stessin M.I. Joint spectra of Toeplitz operators and optimal recovery of analytic functions. Constr. Approx., 2012, vol. 36, no. 1, pp. 53-82. doi: 10.1007/s00365-012-9169-8.

11. DeGraw A. Optimal recovery of holomorphic functions from inaccurate information about radial integration. Amer. J. Comput. Math., 2012, vol. 2, no. 4, pp. 258-268. doi: 10.4236/ajcm.2012.24035.

12. Lavrent'ev M.M., Romanov V.G., Shishatskii S.P. Nekorrektnye zadachi matematicheskoj fiziki i analiza (III-posed problems of mathematical physics and analysis). Providence: American Math. Soc., 1986, Ser. Transl. Math. Monographs, vol. 64, 290 p. 
13. Magaril-Il'yaev G.G., Osipenko K.Yu. Optimal recovery of functionals based on inaccurate data. Math. Notes, 1991, vol. 50, no. 6, pp. 1274-1279. doi: 10.1007/BF01158269.

14. Micchelli Ch.A., Rivlin Th.J. A survey of optimal recovery. In: Optimal estimation in approximation theory. N.Y. etc.: Plenum Press, 1977, pp. 1-54. doi: 10.1007/978-1-4684-2388-4_1.

15. Magaril-Il'yaev G.G., Tikhomirov V.M., Osipenko K.Yu. Indefinite knowledge about an object and accuracy of its recovery methods. Probl. Inf. Transm., 2003, vol. 39, no. 1, pp. 104-118. doi: 10.1023/A:1023686600253.

16. Osipenko K.Yu. Optimal Recovery of Analytic Functions. Huntington: NOVA Science Publ.Inc., 2000, 229 p. ISBN: 1-56072-821-3.

17. Osipenko K.Yu. The Hardy-Littlewood-Polya inequality for analytic functions in Hardy-Sobolev spaces. Sb. Math., 2006, vol. 197, no. 3, pp. 315-334. doi: 10.1070/SM2006v197n03ABEH003760.

18. Osipenko K.Y., Stessin M.I. Hadamard and Schwarz type theorems and optimal recovery in spaces of analytic functions. Constr. Approx., 2010, vol. 31, pp. 37-67. doi: 10.1007/s00365-009-9043-5 .

19. Osipenko K.Yu. Optimal recovery of linear operators in non-Euclidean metrics. Sb. Math., 2014, vol. 205, no. 10, pp. 1442-1472. doi: 10.1070/SM2014v205n10ABEH004425.

Received August 12, 2018

Revised November 14, 2018

Accepted November 19, 2018

Funding Agency: This work was supported by the Russian Foundation for Basic Research (project no. 18-01-00336) and by the Russian Academic Excellence Project (agreement no. 02.A03.21.0006 of August 27, 2013, between the Ministry of Education and Science of the Russian Federation and Ural Federal University).

Roman Razmikovich Akopyan, Ural Federal University, Yekaterinburg, 620000 Russia; Krasovskii Institute of Mathematics and Mechanics, Ural Branch of the Russian Academy of Sciences, Yekaterinburg, 620990 Russia, e-mail: RRAkopyan@mephi.ru . 